\title{
Non-coding RNAs mediate chemotherapy resistance in ovarian
}

\author{
cancer \\ Yang Shao ${ }^{1,2}$, Hui $\mathrm{Li}^{2,3}$, Ran $\mathrm{Du}^{2,3}$, Jiao Meng ${ }^{1,2}$, Gong Yang ${ }^{1,2,3,+}$ \\ ${ }^{1}$ Cancer Institute, Fudan University Shanghai Cancer Center; \\ ${ }^{2}$ Department of Oncology, Shanghai Medical College, Fudan University, Shanghai 200032, China; \\ ${ }^{3}$ Central Laboratory, The Fifth People's Hospital of Shanghai Fudan University, Shanghai 200240, \\ China
}

+ Corresponding Author: E-mail: yanggong@fudan.edu.cn

\begin{abstract}
:
With the advancement of next generation sequencing in past several years, a rising number of non-coding RNAs have been found as new actors to regulate gene expression. Non-coding RNAs not only play important roles in carcinogenesis, but also affect the clinical treatment strategies. They have been proved to be deeply correlated with chemoresistance in several cancers. Ovarian cancer is the leading cause of death in gynecological malignancy, with low 5-year survival rate. Most patients are identified when they have late-stage disease. This review mainly makes a compilation of the most relevant research literature in this field with the purpose of shedding light on the relation between ncRNAs and chemoresistance in ovarian cancer.
\end{abstract}

Key words: ncRNAs; ovarian cancer; chemoresistance 


\section{Introduction}

As one of the most malignant cancers, ovarian cancer is the leading cause of death in gynecological malignancy, especially for epithelial ovarian cancer[1]. The 5-year survival rate of ovarian cancer ranges from $30 \%$ to $90 \%$. In total cases, over $90 \%$ of ovarian cancers are classified as epithelial ovarian cancer. The survival rate is usually close to $90 \%$ at early stages, while a large number of patients are diagnosed as late-stage disease[2,3]. At present, the diagnostic tools mainly involve pelvic examination, transvaginal ultrasound, and serum cancer antigen 125 (CA125) measurements, and the standard therapy is surgery combined with neo adjuvant chemotherapy[4]. Over the past decade, although major advances in the understanding of ovarian cancer have been achieved, tough early diagnosis and poor prognosis still exist. A large number of studies have revealed that non-coding RNAs (ncRNAs) are deeply involved in ovarian cancer progression, including chemoresistance[5,6].

With the development of next-generation sequencing (NGS), chemotherapy resistance has been closely related with nucleic acid, including both DNA and RNA. Besides, based on present studies, ncRNAs have been proved to play an important role in chemotherapy resistance, and even may be the solution of this problem in the future[7,8].

NcRNAs have been proved to regulate many biological processes, including affecting cancer prognosis and influencing response to chemotherapy. The variant expression of ncRNAs in chemotherapy-sensitive versus chemotherapy-resistant cells suggests that modulation of ncRNA expression may reduce or reverse chemotherapy resistance[9-11]. Through participating in several signaling pathways, such as TGF-b/SMAD[12] and p53 signaling pathway[13], ncRNAs can function as potential prognostic biomarker and diagnostic tool in ovarian cancer.

\section{2. ncRNAs and chemoresistance}

Chemoresistance, classified as intrinsic and acquired, is common in chemotherapy. It can accelerate cancer progression and promote morality. Intrinsic 
resistance exists in certain chemo-naive patients, which causes the ineffectiveness of intended chemotherapy. During the course of treatment, acquired resistance can emerge when tumors are resistant to a particular drug[7]. NcRNAs are RNA molecules that are not translated into protein, including highly abundant and functionally important RNAs such as transfer RNAs (tRNAs) and ribosomal RNAs (rRNAs), as well as microRNAs, IncRNAs, circRNAs and tRFs[14]. Large numbers of transcriptomic and bioinformatic studies suggest the existence of thousands of ncRNAs (Figure 1).

Optimal treatment of advanced ovarian cancer includes both surgical cytoreduction and platinum-based chemotherapy. A study demonstrated that patients with stages III and IV ovarian cancer who received chemotherapy had noninferior survival rate and less treatment-related mortality compared with those who received primary cytoreductive surgery[15]..

As one of the critical problems in ovarian cancer therapy, nearly $20 \%$ of all ovarian cancer patients are resistant to the standard platinum-based chemotherapy. Patients treated with chemotherapy will experience relapse and develop drug resistance, and the acquired resistance often faces with no other effective curative treatment[16,17].

\subsection{MicroRNAs}

MicroRNAs (miRNAs) are small non-coding, endogenous, 20-23 nt[18], single-stranded RNAs that regulate gene expression by binding to the 3'-untranslated region (3'-UTR) of mRNAs at post-transcriptional level[19]. A myriad of important biological processes are profoundly influenced by miRNAs, including growth, differentiation, apoptosis, motility, and malignant transformation[20,21].

As key point of many studies, compared with other ncRNAs, the function and regulation mechanism of miRNAs should be much clearer. Bioinformatics data show that miRNAs can regulate at least $20 \%$ to $30 \%$ of human genes. A single miRNA can regulate up to 100 different kinds of mRNAs, while more than 1000 kinds of mRNAs 
can be regulated by the same miRNA[22,23]. An expression profiling study in a group of cisplatin-, paclitaxel-, and cyclosporin A-resistant ovarian cancer cells showed the expressions of let-7e, miR-30c, miR-125b, miR-130a and miR-335 in all resistant cell lines[24].

In a series of studies, miRNAs has been verified to be related with chemotherapy resistance in ovarian cancer. Upregulation of miR-106a and downregulation of miR-591 were associated with paclitaxel resistance in ovarian cancer cells and human tumour samples. Transfection with anti-miR-106a or pre-miR-591 could resensitize paclitaxel-resistant cells to paclitaxel by enhancing apoptosis, and inhibited cell migration and proliferation[25]. Six miRNAs, including miR-23b, miR-27a, miR-27b, miR-346, miR-424, and miR-503, were overexpressed in ovarian ALDH1-positive cells, and they were significantly upregulated in chemoresistant ovarian cancer cells and tumor samples compared with chemosensitive group. High ALDH1 expression was associated with chemoresistance in in vitro and ex vivo samples[26]. Besides, miR-200 family[27-30], miR-199/214 cluster[31-35], miR-31[36] et al, are also reported to be connected with chemotherapy resistance, including cisplatin and pactitaxel (Table 1).

Table 1: MicroRNAs and chemotherapy resistance in ovarian cancer

\begin{tabular}{cccc}
\hline miRNAs & Target genes & Resistance & Reference \\
\hline miR-106a & RB1, p21, BCL10, Caspase-7 & Paclitaxel resistance & {$[25]$} \\
miR-591 & ZEB1 & Paclitaxel resistance & {$[25]$} \\
miR-23b & ALDH1 & Chemoresistance & {$[26]$} \\
miR-27a & ALDH1 & Chemoresistance & {$[26]$} \\
miR-27b & ALDH1 & Chemoresistance & {$[26]$} \\
miR-346 & ALDH1 & Chemoresistance \\
miR-424 & ALDH1 & Chemoresistance
\end{tabular}


miR-503

miR-141
miR-200 family $\quad$ miR-141, miR-200a
miR-200c

miR-199a-5p
miR-199a-3p
miR-199/214 cluster $\quad$ miR-214

miR-31

miR-21
ALDH1

KEAP1

MAPK14

ZEB1, TUBB3

IKK $\beta$, HIF-1A, HIF-2A

CD44

JAG1

PTEN, CCL5

MET

PDCD4
Chemoresistance

Cisplatin resistance

Pactitaxel sensitivity

Pactitaxel sensitivity

Chemosensitivity

Chemosensitivity

Cisplatin sensitivity

Cisplatin resistance

Paclitaxel sensitivity

[36]

[26]

[32,33]

Carboplatin resistance

\subsection{LncRNAs}

Long non-coding RNAs (lncRNAs) is a kind of single-stranded RNA molecule with the length of over $200 \mathrm{nt}$. lncRNAs have been found to exist in every branch of life with organismal complexity, which is better correlated with the diversity and size of ncRNAs expression repertoires than with that of protein-coding genes. lncRNAs engage in numerous important biological processes such as imprinting genomic loci, shaping chromosome conformation and allosterically regulating enzymatic activity. Based on the location, lncRNAs can be classified into sense lncRNAs, anti-sense lncRNAs, intronic lncRNAs, intergenic lncRNAs and divergent lncRNAs. IncRNAs were initially discovered as mRNA-like transcripts that do not encode proteins, but further studies revealed that features of lncRNAs distinguish them from mRNAs. Specific expression of lncRNAs is also proved to coordinate cell state and differentiation. The overexpression, deficiency and mutant of IncRNAs has been found in a series of human diseases.

As a classical lncRNAs, HOX antisense intergenic RNA (HOTAIR) was found to link DNA damage response and chemoresistance in ovarian cancer[38]. This process could also cause the activation $\mathrm{NF}-\kappa \mathrm{B}$ signaling pathway. In related study, upregulation of HOTAIR could induce platinum resistance in ovarian cancer, and 
increased HOTAIR levels were observed in recurrent platinum-resistant ovarian tumors vs. primary ovarian tumors. This suggested that a NF- $\mathrm{KB}-\mathrm{HOTAIR}$ axis drives a positive-feedback loop cascade during DNA damage response and contributes to chemotherapy resistance in ovarian and other cancers[39].

BC200 was also reported to be associated with ovarian cancer. It was observed that carboplatin could upregulate the expression of BC200 in cell lines, and that the inhibition of BC200 decreased the sensitivity of the cells to the drug. So, BC200 appears to serve a role in the mediation of carboplatin-induced ovarian cancer cell death[40].

\subsection{CircRNAs}

Circular RNAs (circRNAs) were discovered as a special novel type of endogenous non-coding RNAs, and have been a new research hotspot in recent years. Different from linear RNAs, circRNAs form a covalently closed continuous loop and are highly represented in the eukaryotic transcriptome. Some studies have demonstrated that thousands of endogenous circRNAs exist in mammalian cells, and most of them are generated from exonic or intronic sequences. In the process of circRNAs biogenesis, RNA-binding proteins (RBPs) are necessary which can regulate circRNAs formation[41-46].

Recent studies revealed that the biological function of circRNAs is abundant. They can affect alternative splicing of transcripts, regulate translation process of some mRNAs, modulate the expression of parental genes, and even function as miRNAs sponges[47-49]. Some reports found that circRNAs are widely involved in diseases, including atherosclerotic vascular disease, neurological disorders, prion diseases and cancers[50-52]. This means, in the near future, circRNAs can be diagnostic or prognostic biomarkers in the clinical treatment.

CircRNAs have recently been identified as miRNA sponges and involve in various biological processes. Limited research showed that circRNAs play some roles in the radioresistance of esophageal cancer. Reported by Wu et al, among the detected 
candidate 3752 circRNAs, significant upregulation of 57 kinds and downregulation of 17 kinds in human were observed in radioresistant esophageal cancer cell line. CircRNA_001059 and circRNA_000167 were the two largest nodes in circRNA/miRNA co-expression network[53]. Although no studies have verified the relationship between circRNAs and chemoresistance, circRNAs are widely involved in many biological processes and interact with miRNAs, which means that circRNAs may also affect the chemoresistance in cancer.

\section{5 tRFs}

tRNA-derived RNA fragments (tRFs) is a class of non-coding single-stranded RNA with the length of 14-35 nt, which always derive from 5' end or 3' end of tRNAs in the particular environment [54]. Some tRNA cleavages can be generated from mature tRNAs with the length of 30-35 nt, which are called tRNA halves. The rest of tRNA cleavages can be classified into three groups: 3' U tRFs, 3' CCA tRFs and 5' tRFs. 3' U tRFs can be generated during the maturation of tRNA in the nucleus, while 3' CCA tRFs and 5' tRFs can be generated at any time from mature tRNAs $[55,56]$.

Up to now, it hasn't been found any direct relationship between tRFs and chemotherapy resistance. However, tRFs have been proved to be closely connected with stress condition[57]. Chemotherapy resistance or sensitivity is much likely to be associated with cancer microenvironment, and then affect the stress condition in vivo. This means, due to the change of microenvironment, tRFs may play a key role in chemotherapy resistance, which is believed to be the research hotspot in the future.

\section{The crosstalk between ncRNAs in ovarian cancer}

The transcription process is completed in vivo, from DNA to RNA, and as the first step of proteins biosynthesis, it is also the synthesis procedure of mRNAs and other ncRNAs (tRNAs, rRNAs, etc).

As the most abundant RNAs, tRNAs occupy nearly $15 \%$ of total RNAs in 
humans. A large amount of reports show that tRNAs can be cleaved into small molecule fragments under certain stress condition, called tRFs. At present, the biological function of tRFs still keeps unclear, and limited research indicate tRFs have a close relationship with RNA silence and Argonaute family proteins. Besides, tRFs were once found to regulate the progression of breast cancer via YBX1[58].

Compared to tRFs, the mechanism of miRNAs and lncRNAs is much clearer. Through binding to the 3'-untranslated region (3'-UTR) of mRNAs, miRNAs can cause mRNA degradation and inhibit translation. In many signaling pathways, miRNAs also play important roles, and then have close correlation with critical diseases[59-61].

As a kind of typical ncRNAs, lncRNAs can be combined with both mRNAs and miRNAs, as well as perform gene silencing on them. The latest research shows the new function of lncRNAs that they can encode some proteins in organisms, which is different from traditional research[62].

XIST RNAs are a classical kind of lncRNAs, whose model for gene silencing mechanism has been established. Based on its own three-dimensional structure, $\mathrm{X}$ chromosome can bind XIST RNA to the nearby binding sites, and in turn XIST RNA can change the structure of $\mathrm{X}$ chromosome, which eventually makes $\mathrm{X}$ chromosome silent.

CircRNAs are a new class of RNAs which is different from traditional linear RNAs. It has a closed loop structure and is abundant in eukaryotic transcription. Most of circRNAs are composed of exon sequences, which are conserved in different species and show expression specificity in different tissues and developmental stages. Since circRNAs aren't sensitive to nuclease, they are more stable than linear RNAs. Recent studies reveal that circRNAs and lncRNAs are both competing endogenous RNAs (ceRNA), and can compete with microRNA response elements (MREs) to regulate the expression of transcriptosome. CircRNAs can affect miRNAs directly or indirectly, and then function as miRNAs sponges to mediate target genes. This means circRNAs may play key roles in chemotherapy resistance through the interaction with $\operatorname{miRNAs}[63,64]$. 


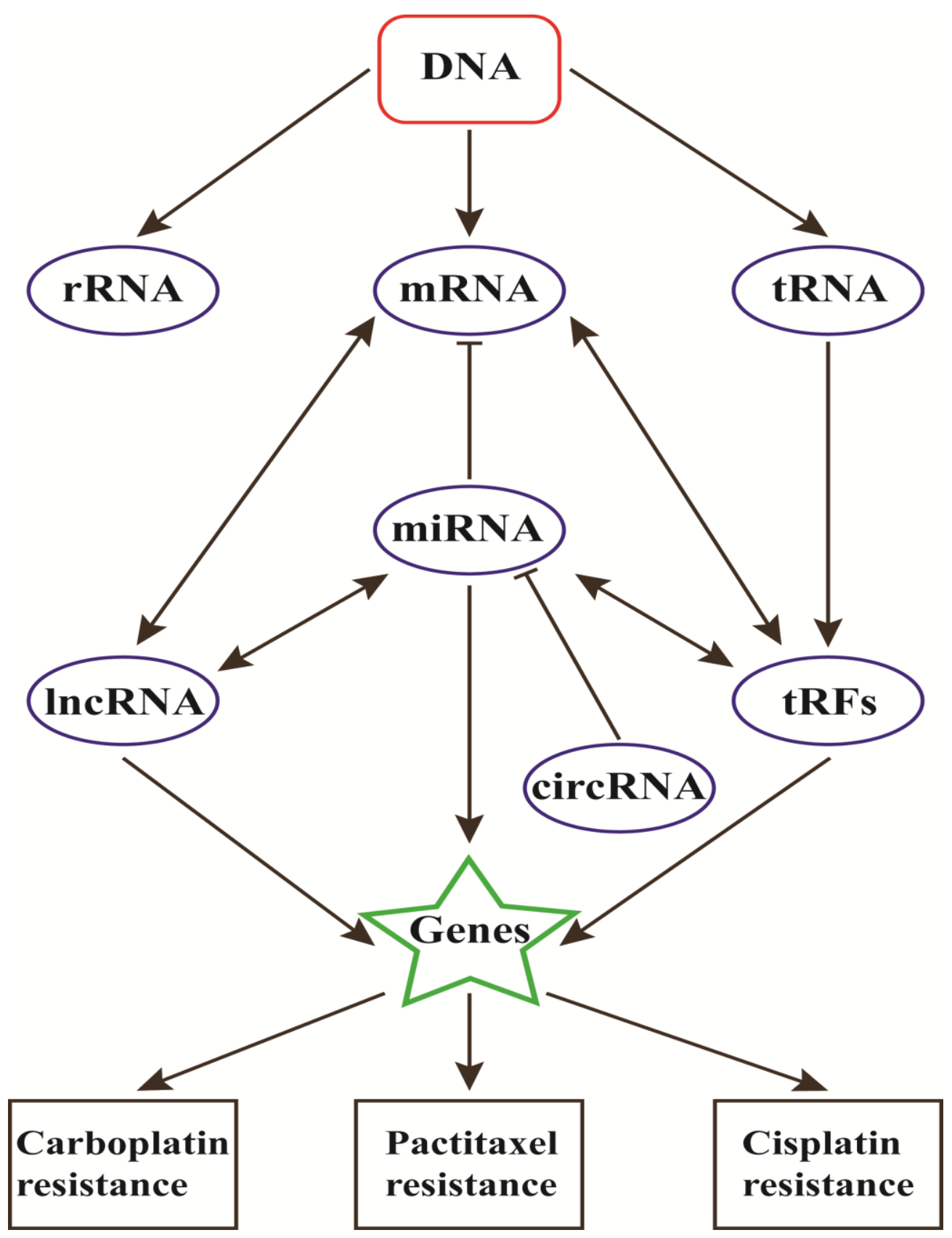

Figure 1: The relationship between ncRNAs and chemotherapy resistance in ovarian cancer

\section{Chemoresistance and precision medicine}

The development of modern medical technology is gradually revealing the mechanisms of critical diseases. Cancer is thought as a 'genetic disease', and with the advancement of NGS and HGP, the therapeutic strategy for cancer has gradually moved towards the era of precision medical. In this context, the roles of RNAs will become much more important.

Among of all ncRNAs, miRNAs have the most potential to be the diagnostic 
biomarker and therapeutic drug. For example, as the most famous miRNAs, miR-34 play important roles in breast cancer and ovarian cancer, through targeting EGFR, Bcl-2, CCND1 and so on[65-67]. Compared with other ncRNAs, the mechanisms of miRNAs are much clearer, whose biological function is performed through participating in several signaling pathways. The abundant features make it possible for miRNAs to be key members in precision medical.

As a kind of novel ncRNAs, the research on tRFs is still at start stage. Since the similarity with miRNAs in structure, tRFs were once thought as one kind of miRNAs. Besides, the generation of tRFs is closely related with stress condition, which means they are more sensitive to cancer microenvironment, and it is more possible for them to be diagnostic biomarker.

The reports that circRNAs can function as ceRNA make it to be hotspots in the research field of cancers. Because of closed loop structure, circRNAs can be away from the effect of exonuclease. Due to its stability, the existence of circRNAs in cells and human blood is long-term. In this circumstance, circRNAs are thought as the most suitable early diagnostic biomarker by some researchers.

Based on the unique features, lncRNAs can be combined with other ncRNAs and mRNAs, functioning as gene silencing. The biological mechanism of lncRNAs is full of complexity, and they can even encode proteins. In many instances, lncRNAs can be the bridge of the crosstalk between other ncRNAs.

With the advancement of precision medical, the relationship between ncRNAs and diseases will be the research focus, including cancers and chemotherapy resistance. The total kinds of ncRNAs have researched new heights, but it is believed that there still exists other types of ncRNAs, and they can form a RNA world.

\section{Future perspectives}

ncRNAs have myriad roles in cells, from controlling the architecture of whole chromosomes and acting as a regulator of gene expression to affecting the translation of genetic codons into protein sequences. With the help of different features, including 
unique regulatory mechanisms, alternative forms of biogenesis and functional structured RNA domains, ncRNAs can be classified into several types, and distinguished from each other. Current deep RNA-sequencing and advanced epigenomic technologies are accelerating the rate of discovering new ncRNAs, this will help us to characterize them. Moving forward, with these and other future techniques we will surely uncover even more fascinating and unique features and functions of ncRNAs. It can be expected that ncRNA-based therapeutics will play an important role in the future of clinical medicine[68].

Based on present studies, miRNAs have the most abundant functions in ovarian cancer chemoresistance. Several miRNAs have been identified to be remarkably up/down-regulated in chemoresistant ovarian cancer, including let-7i, miR-27a, miR-23a, miR-449b, miR-21 and so on[69]. They are associated with poor prognosis and short survival in chemoresistant ovarian cancer patients. Besides, lncRNA also play key roles in chemresistance. They are closely linked with DNA damage and repair. Contrast to miRNAs and lncRNAs, the biological function of circRNAs and tRFs in chemoresistance still keeps unclear. In view of their relationship with miRNAs and lncRNAs, they are also believed to contribute to chemoresistance in ovarian cancer[70].

Although many breakthroughs have been made on regulatory mechanism of sncRNAs, a significant number of them remain unknown. For example, tRFs were once thought of as a kind of miRNAs, but later, they were affirmed to be a type of cleavage product from tRNAs, different from miRNAs. So, for us, it is still necessary to be absorbed into the studies of ncRNAs, just not only to correct any possible mistakes, but also to get more achievements for the development of physianthropy. 


\section{References:}

1. Chen, W.; Zheng, R.; Baade, P.D.; Zhang, S.; Zeng, H.; Bray, F.; Jemal, A.; Yu, X.Q.; He, J. Cancer statistics in china, 2015. CA: a cancer journal for clinicians 2016.

2. Di Leva, G.; Croce, C.M. Mirna profiling of cancer. Current opinion in genetics \& development 2013, 23, 3-11.

3. Prat, J.; Oncology, F.C.o.G. Staging classification for cancer of the ovary, fallopian tube, and peritoneum. International journal of gynaecology and obstetrics: the official organ of the International Federation of Gynaecology and Obstetrics 2014, 124, 1-5.

4. $\quad$ van Haaften-Day, C.; Shen, Y.; Xu, F.; Yu, Y.; Berchuck, A.; Havrilesky, L.J.; de Bruijn, H.W.; van der Zee, A.G.; Bast, R.C., Jr.; Hacker, N.F. Ovx1, macrophage-colony stimulating factor, and ca-125-ii as tumor markers for epithelial ovarian carcinoma: A critical appraisal. Cancer 2001, 92, 2837-2844.

5. Redman, C.; Duffy, S.; Bromham, N.; Francis, K.; Guideline Development, G. Recognition and initial management of ovarian cancer: Summary of nice guidance. Bmj 2011, 342, d2073.

6. Karlan, B.Y.; Alvarez, R.D.; Strauss, J.F., 3rd. Evolving paradigms in research and care in ovarian cancers. Obstetrics and gynecology 2016, 128, 771-774.

7. Frederick, P.J.; Green, H.N.; Huang, J.S.; Egger, M.E.; Frieboes, H.B.; Grizzle, W.E.; McNally, L.R. Chemoresistance in ovarian cancer linked to expression of micrornas. Biotechnic \& histochemistry : official publication of the Biological Stain Commission 2013, 88, 403-409.

8. Xing, Z.; Li, D.; Yang, L.; Xi, Y.; Su, X. Micrornas and anticancer drugs. Acta biochimica et biophysica Sinica 2014, 46, 233-239.

9. Meyer, L.A.; Cronin, A.M.; Sun, C.C.; Bixel, K.; Bookman, M.A.; Cristea, M.C.; Griggs, J.J.; Levenback, C.F.; Burger, R.A.; Mantia-Smaldone, G., et al. Use and effectiveness of neoadjuvant chemotherapy for treatment of ovarian cancer. Journal of clinical oncology : official journal of the American Society of Clinical Oncology 2016.

10. Zeng, L.J.; Xiang, C.L.; Gong, Y.Z.; Kuang, Y.; Lu, F.F.; Yi, S.Y.; Zhang, Y.; Liao, M. Neoadjuvant chemotherapy for patients with advanced epithelial ovarian cancer: A meta-analysis. Scientific reports 2016, 6, 35914.

11. Egloff, H.; Jatoi, A. Do ovarian cancer patients with a family history of cancer (suspected brca1 or brca2 mutation) suffer greater chemotherapy toxicity? Cancer investigation 2016, $1-5$.

12. Parikh, A.; Lee, C.; Joseph, P.; Marchini, S.; Baccarini, A.; Kolev, V.; Romualdi, C.; Fruscio, R.; Shah, H.; Wang, F., et al. Microrna-181a has a critical role in ovarian cancer progression through the regulation of the epithelial-mesenchymal transition. Nature communications $\mathbf{2 0 1 4}$, $5,2977$.

13. Zhang, L.; Volinia, S.; Bonome, T.; Calin, G.A.; Greshock, J.; Yang, N.; Liu, C.G.; Giannakakis, A.; Alexiou, P.; Hasegawa, K., et al. Genomic and epigenetic alterations deregulate microrna expression in human epithelial ovarian cancer. Proceedings of the National Academy of Sciences of the United States of America 2008, 105, 7004-7009.

14. Kawaji, H.; Nakamura, M.; Takahashi, Y.; Sandelin, A.; Katayama, S.; Fukuda, S.; Daub, C.O.; Kai, C.; Kawai, J.; Yasuda, J., et al. Hidden layers of human small rnas. BMC genomics 2008, $9,157$.

15. Vergote, I.; Trope, C.G.; Amant, F.; Kristensen, G.B.; Ehlen, T.; Johnson, N.; Verheijen, R.H.; 
van der Burg, M.E.; Lacave, A.J.; Panici, P.B., et al. Neoadjuvant chemotherapy or primary surgery in stage iiic or iv ovarian cancer. The New England journal of medicine 2010, 363, 943-953.

16. Prahm, K.P.; Novotny, G.W.; Hogdall, C.; Hogdall, E. Current status on micrornas as biomarkers for ovarian cancer. APMIS : acta pathologica, microbiologica, et immunologica Scandinavica 2016, 124, 337-355.

17. Weinberg, L.E.; Rodriguez, G.; Hurteau, J.A. The role of neoadjuvant chemotherapy in treating advanced epithelial ovarian cancer. Journal of surgical oncology 2010, 101, 334-343.

18. Oom, A.L.; Humphries, B.A.; Yang, C. Micrornas: Novel players in cancer diagnosis and therapies. BioMed research international 2014, 2014, 959461.

19. Bartel, D.P. Micrornas: Target recognition and regulatory functions. Cell 2009, 136, 215-233.

20. Garzon, R.; Calin, G.A.; Croce, C.M. Micrornas in cancer. Annual review of medicine 2009, 60, 167-179.

21. Xu, X.; Chen, W.; Miao, R.; Zhou, Y.; Wang, Z.; Zhang, L.; Wan, Y.; Dong, Y.; Qu, K.; Liu, C. Mir-34a induces cellular senescence via modulation of telomerase activity in human hepatocellular carcinoma by targeting foxm1/c-myc pathway. Oncotarget 2015, 6, 3988-4004.

22. Santulli, G. Exploiting microrna specificity and selectivity: Paving a sustainable path towards precision medicine. Advances in experimental medicine and biology 2015, 888, 1-3.

23. Bertoli, G.; Cava, C.; Castiglioni, I. Micrornas: New biomarkers for diagnosis, prognosis, therapy prediction and therapeutic tools for breast cancer. Theranostics 2015, 5, 1122-1143.

24. Sorrentino, A.; Liu, C.G.; Addario, A.; Peschle, C.; Scambia, G.; Ferlini, C. Role of micrornas in drug-resistant ovarian cancer cells. Gynecologic oncology 2008, 111, 478-486.

25. Huh, J.H.; Kim, T.H.; Kim, K.; Song, J.A.; Jung, Y.J.; Jeong, J.Y.; Lee, M.J.; Kim, Y.K.; Lee, D.H.; An, H.J. Dysregulation of mir-106a and mir-591 confers paclitaxel resistance to ovarian cancer. British journal of cancer 2013, 109, 452-461.

26. Park, Y.T.; Jeong, J.Y.; Lee, M.J.; Kim, K.I.; Kim, T.H.; Kwon, Y.D.; Lee, C.; Kim, O.J.; An, H.J. Micrornas overexpressed in ovarian aldh1-positive cells are associated with chemoresistance. Journal of ovarian research 2013, 6, 18.

27. van Jaarsveld, M.T.; Helleman, J.; Boersma, A.W.; van Kuijk, P.F.; van Ijcken, W.F.; Despierre, E.; Vergote, I.; Mathijssen, R.H.; Berns, E.M.; Verweij, J., et al. Mir-141 regulates keap1 and modulates cisplatin sensitivity in ovarian cancer cells. Oncogene 2013, 32, 4284-4293.

28. Cittelly, D.M.; Dimitrova, I.; Howe, E.N.; Cochrane, D.R.; Jean, A.; Spoelstra, N.S.; Post, M.D.; Lu, X.; Broaddus, R.R.; Spillman, M.A., et al. Restoration of mir-200c to ovarian cancer reduces tumor burden and increases sensitivity to paclitaxel. Molecular cancer therapeutics 2012, 11, 2556-2565.

29. Cochrane, D.R.; Spoelstra, N.S.; Howe, E.N.; Nordeen, S.K.; Richer, J.K. Microrna-200c mitigates invasiveness and restores sensitivity to microtubule-targeting chemotherapeutic agents. Molecular cancer therapeutics 2009, 8, 1055-1066.

30. Mateescu, B.; Batista, L.; Cardon, M.; Gruosso, T.; de Feraudy, Y.; Mariani, O.; Nicolas, A.; Meyniel, J.P.; Cottu, P.; Sastre-Garau, X., et al. Mir-141 and mir-200a act on ovarian tumorigenesis by controlling oxidative stress response. Nature medicine 2011, 17, 1627-1635.

31. Cheng, W.; Liu, T.; Wan, X.; Gao, Y.; Wang, H. Microrna-199a targets cd44 to suppress the tumorigenicity and multidrug resistance of ovarian cancer-initiating cells. The FEBS journal 2012, 279, 2047-2059. 
32. Joshi, H.P.; Subramanian, I.V.; Schnettler, E.K.; Ghosh, G.; Rupaimoole, R.; Evans, C.; Saluja, M.; Jing, Y.; Cristina, I.; Roy, S., et al. Dynamin 2 along with microrna-199a reciprocally regulate hypoxia-inducible factors and ovarian cancer metastasis. Proceedings of the National Academy of Sciences of the United States of America 2014, 111, 5331-5336.

33. Chen, R.; Alvero, A.B.; Silasi, D.A.; Kelly, M.G.; Fest, S.; Visintin, I.; Leiser, A.; Schwartz, P.E.; Rutherford, T.; Mor, G. Regulation of ikkbeta by mir-199a affects nf-kappab activity in ovarian cancer cells. Oncogene 2008, 27, 4712-4723.

34. Liu, M.X.; Siu, M.K.; Liu, S.S.; Yam, J.W.; Ngan, H.Y.; Chan, D.W. Epigenetic silencing of microrna-199b-5p is associated with acquired chemoresistance via activation of jag1-notch1 signaling in ovarian cancer. Oncotarget 2014, 5, 944-958.

35. Yang, H.; Kong, W.; He, L.; Zhao, J.J.; O'Donnell, J.D.; Wang, J.; Wenham, R.M.; Coppola, D.; Kruk, P.A.; Nicosia, S.V., et al. Microrna expression profiling in human ovarian cancer: Mir-214 induces cell survival and cisplatin resistance by targeting pten. Cancer research $\mathbf{2 0 0 8}$, $68,425-433$.

36. Mitamura, T.; Watari, H.; Wang, L.; Kanno, H.; Hassan, M.K.; Miyazaki, M.; Katoh, Y.; Kimura, T.; Tanino, M.; Nishihara, H., et al. Downregulation of mirna-31 induces taxane resistance in ovarian cancer cells through increase of receptor tyrosine kinase met. Oncogenesis 2013, 2, e40.

37. Ren, W.; Wang, X.; Gao, L.; Li, S.; Yan, X.; Zhang, J.; Huang, C.; Zhang, Y.; Zhi, K. Mir-21 modulates chemosensitivity of tongue squamous cell carcinoma cells to cisplatin by targeting pdcd4. Molecular and cellular biochemistry 2014, 390, 253-262.

38. Li, J.; Yang, S.; Su, N.; Wang, Y.; Yu, J.; Qiu, H.; He, X. Overexpression of long non-coding rna hotair leads to chemoresistance by activating the wnt/beta-catenin pathway in human ovarian cancer. Tumour biology : the journal of the International Society for Oncodevelopmental Biology and Medicine 2016, 37, 2057-2065.

39. Ozes, A.R.; Miller, D.F.; Ozes, O.N.; Fang, F.; Liu, Y.; Matei, D.; Huang, T.; Nephew, K.P. Nf-kappab-hotair axis links DNA damage response, chemoresistance and cellular senescence in ovarian cancer. Oncogene 2016, 35, 5350-5361.

40. Wu, D.I.; Wang, T.; Ren, C.; Liu, L.; Kong, D.; Jin, X.; Li, X.; Zhang, G. Downregulation of bc200 in ovarian cancer contributes to cancer cell proliferation and chemoresistance to carboplatin. Oncology letters 2016, 11, 1189-1194.

41. Lasda, E.; Parker, R. Circular rnas: Diversity of form and function. Rna 2014, 20, 1829-1842.

42. Conn, S.J.; Pillman, K.A.; Toubia, J.; Conn, V.M.; Salmanidis, M.; Phillips, C.A.; Roslan, S.; Schreiber, A.W.; Gregory, P.A.; Goodall, G.J. The rna binding protein quaking regulates formation of circrnas. Cell 2015, 160, 1125-1134.

43. Zhang, Y.; Zhang, X.O.; Chen, T.; Xiang, J.F.; Yin, Q.F.; Xing, Y.H.; Zhu, S.; Yang, L.; Chen, L.L. Circular intronic long noncoding rnas. Molecular cell 2013, 51, 792-806.

44. Li, Z.; Huang, C.; Bao, C.; Chen, L.; Lin, M.; Wang, X.; Zhong, G.; Yu, B.; Hu, W.; Dai, L., et al. Exon-intron circular rnas regulate transcription in the nucleus. Nature structural \& molecular biology 2015, 22, 256-264.

45. Jeck, W.R.; Sorrentino, J.A.; Wang, K.; Slevin, M.K.; Burd, C.E.; Liu, J.; Marzluff, W.F.; Sharpless, N.E. Circular rnas are abundant, conserved, and associated with alu repeats. Rna 2013, 19, 141-157.

46. Salzman, J.; Chen, R.E.; Olsen, M.N.; Wang, P.L.; Brown, P.O. Cell-type specific features of 
circular rna expression. PLoS genetics 2013, 9, e1003777.

47. Jeck, W.R.; Sharpless, N.E. Detecting and characterizing circular rnas. Nature biotechnology 2014, 32, 453-461.

48. Ashwal-Fluss, R.; Meyer, M.; Pamudurti, N.R.; Ivanov, A.; Bartok, O.; Hanan, M.; Evantal, N.; Memczak, S.; Rajewsky, N.; Kadener, S. Circrna biogenesis competes with pre-mrna splicing. Molecular cell 2014, 56, 55-66.

49. Kulcheski, F.R.; Christoff, A.P.; Margis, R. Circular rnas are mirna sponges and can be used as a new class of biomarker. Journal of biotechnology 2016, 238, 42-51.

50. Maiese, K. Disease onset and aging in the world of circular rnas. Journal of translational science 2016, 2, 327-329.

51. Chen, B.J.; Mills, J.D.; Takenaka, K.; Bliim, N.; Halliday, G.M.; Janitz, M. Characterization of circular rnas landscape in multiple system atrophy brain. Journal of neurochemistry 2016, 139, 485-496.

52. Nair, A.A.; Niu, N.; Tang, X.; Thompson, K.J.; Wang, L.; Kocher, J.P.; Subramanian, S.; Kalari, K.R. Circular rnas and their associations with breast cancer subtypes. Oncotarget 2016.

53. Su, H.; Lin, F.; Deng, X.; Shen, L.; Fang, Y.; Fei, Z.; Zhao, L.; Zhang, X.; Pan, H.; Xie, D., et al. Profiling and bioinformatics analyses reveal differential circular rna expression in radioresistant esophageal cancer cells. Journal of translational medicine 2016, 14, 225.

54. Lee, Y.S.; Shibata, Y.; Malhotra, A.; Dutta, A. A novel class of small rnas: Trna-derived rna fragments (trfs). Genes \& development 2009, 23, 2639-2649.

55. Thompson, D.M.; Parker, R. Stressing out over trna cleavage. Cell 2009, 138, 215-219.

56. Haiser, H.J.; Karginov, F.V.; Hannon, G.J.; Elliot, M.A. Developmentally regulated cleavage of trnas in the bacterium streptomyces coelicolor. Nucleic acids research 2008, 36, 732-741.

57. Durdevic, Z.; Schaefer, M. Trna modifications: Necessary for correct trna-derived fragments during the recovery from stress? BioEssays : news and reviews in molecular, cellular and developmental biology 2013, 35, 323-327.

58. Goodarzi, H.; Liu, X.; Nguyen, H.C.; Zhang, S.; Fish, L.; Tavazoie, S.F. Endogenous trna-derived fragments suppress breast cancer progression via ybx1 displacement. Cell 2015, $161,790-802$.

59. Ji, X.; Wang, Z.; Geamanu, A.; Goja, A.; Sarkar, F.H.; Gupta, S.V. Delta-tocotrienol suppresses notch-1 pathway by upregulating mir-34a in nonsmall cell lung cancer cells. International journal of cancer. Journal international du cancer 2012, 131, 2668-2677.

60. Liu, Z.L.; Wang, H.; Liu, J.; Wang, Z.X. Microrna-21 (mir-21) expression promotes growth, metastasis, and chemo- or radioresistance in non-small cell lung cancer cells by targeting pten. Molecular and cellular biochemistry 2013, 372, 35-45.

61. Zhang, J.; Lu, Y.; Yue, X.; Li, H.; Luo, X.; Wang, Y.; Wang, K.; Wan, J. Mir-124 suppresses growth of human colorectal cancer by inhibiting stat3. PloS one 2013, 8, e70300.

62. Paraskevopoulou, M.D.; Hatzigeorgiou, A.G. Analyzing mirna-lncrna interactions. Methods in molecular biology 2016, 1402, 271-286.

63. Dong, R.; Zhang, X.O.; Zhang, Y.; Ma, X.K.; Chen, L.L.; Yang, L. Circrna-derived pseudogenes. Cell research 2016, 26, 747-750.

64. Chen, L.L.; Yang, L. Regulation of circrna biogenesis. RNA biology 2015, 12, 381-388.

65. Li, N.; Fu, H.; Tie, Y.; Hu, Z.; Kong, W.; Wu, Y.; Zheng, X. Mir-34a inhibits migration and 
invasion by down-regulation of c-met expression in human hepatocellular carcinoma cells. Cancer letters 2009, 275, 44-53.

66. Li, X.J.; Ji, M.H.; Zhong, S.L.; Zha, Q.B.; Xu, J.J.; Zhao, J.H.; Tang, J.H. Microrna-34a modulates chemosensitivity of breast cancer cells to adriamycin by targeting notch1. Archives of medical research 2012, 43, 514-521.

67. Zhou, J.Y.; Chen, X.; Zhao, J.; Bao, Z.; Chen, X.; Zhang, P.; Liu, Z.F.; Zhou, J.Y. Microrna-34a overcomes hgf-mediated gefitinib resistance in egfr mutant lung cancer cells partly by targeting met. Cancer letters 2014, 351, 265-271.

68. Enfield, K.S.; Martinez, V.D.; Marshall, E.A.; Stewart, G.L.; Kung, S.H.; Enterina, J.R.; Lam, W.L. Deregulation of small non-coding rnas at the dlk1-dio3 imprinted locus predicts lung cancer patient outcome. Oncotarget 2016.

69. Mahdian-Shakib, A.; Dorostkar, R.; Tat, M.; Hashemzadeh, M.S.; Saidi, N. Differential role of micrornas in prognosis, diagnosis, and therapy of ovarian cancer. Biomedicine \& pharmacotherapy = Biomedecine \& pharmacotherapie 2016, 84, 592-600.

70. Ayers, D.; Vandesompele, J. Influence of micrornas and long non-coding rnas in cancer chemoresistance. Genes 2017, 8 . 